Check for updates

Cite this: J. Mater. Chem. A, 2020, 8 , 6360

Received 29th October 2019

Accepted 6th March 2020

DOI: 10.1039/c9ta11912d

rsc.li/materials-a

\section{The role of oxygen vacancies on the vibrational motions of hydride ions in the oxyhydride of barium titanate $\uparrow$}

\author{
Carin Eklöf-Österberg, ${ }^{a}$ Laura Mazzei, (DD b Erik Jedvik Granhed, (DD b \\ Göran Wahnström, (D) ${ }^{\mathrm{b}}$ Reji Nedumkandathil, ${ }^{\mathrm{C}}$ Ulrich Häussermann, ${ }^{\mathrm{c}}$ \\ Aleksander Jaworski, ${ }^{c}$ Andrew J. Pell, (D) ${ }^{c}$ Stewart F. Parker, (D) ${ }^{d}$ Niina H. Jalarvo, (D) \\ Lars Börjesson ${ }^{\mathrm{b}}$ and Maths Karlsson (iD *a
}

Perovskite-type oxyhydrides, $\mathrm{BaTiO}_{3-x} \mathrm{H}_{x}$, represent a novel class of hydride ion conducting materials of interest for several electrochemical applications, but fundamental questions surrounding the defect chemistry and hydride ion transport mechanism remain unclear. Here we report results from powder Xray diffraction, thermal gravimetric analysis, nuclear magnetic resonance spectroscopy, inelastic neutron scattering (INS), and density functional theory (DFT) simulations on three metal hydride reduced $\mathrm{BaTiO}_{3}$ samples characterized by the simultaneous presence of hydride ions and oxygen vacancies. The INS spectra are characterized by two predominating bands at around $114\left(\omega_{\perp}\right)$ and $128\left(\omega_{\|}\right)$meV, assigned as fundamental $\mathrm{Ti}-\mathrm{H}$ vibrational modes perpendicular and parallel to the $\mathrm{Ti}-\mathrm{H}-\mathrm{Ti}$ bond direction, respectively, and four additional, weaker, bands at around $99\left(\omega_{1}\right), 110\left(\omega_{2}\right), 137\left(\omega_{3}\right)$ and $145\left(\omega_{4}\right) \mathrm{meV}$ that originate from a range of different local structures associated with different configurations of the hydride ions and oxygen vacancies in the materials. Crucially, the combined analyses of INS and DFT data confirm the presence of both nearest and next-nearest neighbouring oxygen vacancies to the hydride ions. This supports previous findings from quasielastic neutron scattering experiments, that the hydride ion transport is governed by jump diffusion dynamics between neighbouring and next-nearest neighbouring hydride ion-oxygen vacancy local structures. Furthermore, the investigation of the momentum transfer dependence of the INS spectrum is used to derive the mean square displacement of the hydride ions, which is shown to be in excellent agreement with the calculations. Analysis of the mean square displacement confirms that the hydrogen vibrational motions are localized in nature and only very weakly affected by the dynamics of the surrounding perovskite structure. This insight motivates efforts to identify alternative host lattices that allow for a less localization of the hydride ions as a route to higher hydride ion conductivities.

\section{Introduction}

Perovskite-type oxyhydrides $\mathrm{ATiO}_{3-x} \mathrm{H}_{x}(\mathrm{~A}=\mathrm{Ba}, \mathrm{Sr}, \mathrm{Ca}, x<0.6)$, in which some of the oxide ions are replaced by hydride ions, have

\footnotetext{
${ }^{a}$ Department of Chemistry and Chemical Engineering, Chalmers University of Technology, SE-412 96 Göteborg, Sweden. E-mail: maths.karlsson@chalmers.se; Tel: $+46317726770$

${ }^{b}$ Department of Physics, Chalmers University of Technology, SE-412 96 Göteborg, Sweden

'Department of Materials and Environmental Chemistry, Stockholm University, SE10691 Stockholm, Sweden

${ }^{d}$ ISIS Neutron and Muon Source, STFC Rutherford Appleton Laboratory, Oxfordshire OX11 OQX, UK

${ }^{e}$ Chemical and Engineering Materials Division, Oak Ridge National Laboratory, Oak Ridge, Tennessee 37831-6475, USA

$\dagger$ Electronic supplementary information (ESI) available. See DOI: $10.1039 / \mathrm{c} 9 \operatorname{ta} 11912 \mathrm{~d}$
}

recently emerged as a novel class of mixed electronic-ionic conductors with potential application in energy storage and conversion. ${ }^{\mathbf{1}-\mathbf{3}}$ Understanding the defect chemistry and hydride ion conduction mechanism in these materials is of great importance for developing efficient synthesis routes that allow the development of new oxyhydrides with tailored ionic and electronic conductivity for certain applications, but neither the defect chemistry nor hydride ion conduction mechanism are fully understood. Recent results from quasielastic neutron scattering (QENS), ${ }^{4}$ isotope exchange, ${ }^{5,6}$ combined isotope exchange with depth profiling analysis ${ }^{7}$ and first-principles calculations ${ }^{7,8}$ suggest a vacancy-assisted diffusion mechanism. However, a mechanism based on the transformation into a proton and then interstitial diffusion has been also discussed in the past., ${ }^{\mathbf{1 , - 1 0}}$

The oxyhydrides $\mathrm{ATiO}_{3-x} \mathrm{H}_{x}$ are formed through a topochemical approach by reacting $\mathrm{ATiO}_{3}$ with, e.g., $\mathrm{CaH}_{2}$, which gives rise to the substitution of oxide ions by hydride ions. Among 
these, $\mathrm{BaTiO}_{3-x} \mathrm{H}_{x}$ exhibits the highest amount of hydride ions with a concentration up to $x \approx 0.6$. The substitutional hydride ion acts as a donor, contributing to $\mathrm{n}$ type conductivity in the initially empty $\mathrm{Ti}^{4+} 3 \mathrm{~d}$ band, ${ }^{8-10}$ and conductivity measurements of $\mathrm{BaTiO}_{3-x} \mathrm{H}_{x}$ shows that it is electronically conducting., ${ }^{\mathbf{1 1 1}, 12}$ The conductivities as reported on $\mathrm{BaTiO}_{3-x} \mathrm{H}_{x}$ are, however, somewhat conflicting in that both semiconducting ${ }^{1}$ and metallic-like ${ }^{12}$ conductivity has been reported. The semiconducting behaviour was suggested to relate to the presence of localized electronic states within the bandgap, ${ }^{8}$ which are associated with electron polarons. A polaron in this case refers to when an electron is localized on a titanium ion that resides next to a hydride ion and thereby changes the valence of the titanium ion from +4 to +3 .

Recently, we showed, in a combined density functional theory (DFT) calculations and inelastic neutron scattering (INS) study of $\mathrm{BaTiO}_{3-x} \mathrm{H}_{y} \square_{x-y}$ with $y=0.1$ and $x=0.18$, that the electrons rather tend to form a delocalized state where they are shared among all $\mathrm{Ti}^{4+}$ ions, thus forming a bandstate configuration..$^{13} \mathrm{In}$ particular, the INS technique was used to determine the vibrational spectrum of $\mathrm{BaTiO}_{3-x} \mathrm{H}_{x}$, which was shown to be characterized by two intense peaks at approximately 113 and $128 \mathrm{meV}$, respectively, assigned as vibrational motions of the hydride ions. The DFT calculations showed that these vibrational modes are essentially localized, i.e. show, unlike collective phonons, little dispersion. ${ }^{13}$ This stems from the large mass difference between the hydrogen and the other atoms in the lattice and means that the vibrational motions of the hydride ions can be treated as decoupled from both the vibrational dynamics of the perovskite lattice structure and the vibrational dynamics of other hydride ions. In other words, one can study such vibrations as if they were virtually isolated from the surrounding system. In addition to the main vibrational bands at 113 and $128 \mathrm{meV}$, the INS spectrum of $\mathrm{BaTiO}_{3-x} \mathrm{H}_{x}$ is also characterized by an array of weaker bands at higher frequencies, tentatively assigned as higher-order transitions of the two main vibrational modes. ${ }^{13}$

Here, we report on a study of the local structure and vibrational dynamics of three metal hydride reduced $\mathrm{BaTiO}_{3}$ samples, which are characterized by varying concentrations of hydride ions and oxygen vacancies. The techniques used are powder X-ray diffraction (PXRD), thermal gravimetric analysis (TGA), nuclear magnetic resonance (NMR) spectroscopy, INS, and DFT calculations. Analyses of the intensity and momentum transfer $(Q)$ of the INS spectral bands provide unique information to characterize the local coordination of hydride ions, such as vibrational frequencies and relative occupation of different hydride ion sites, distinguish between fundamental modes and higher-order transitions, and to derive the mean square displacements (MSD) of the hydride ions. The results provide experimental evidence to the fact that the hydride ion vibrational dynamics are localized in nature and only weakly affected by the dynamics of the surrounding perovskite framework.

\section{Experimental details}

\subsection{Sample preparation}

Two reduced $\mathrm{BaTiO}_{3}$ samples, which in the following are termed $\mathrm{CA} 2$ and $\mathrm{CA} 3$, were prepared by mixing powders of $\mathrm{BaTiO}_{3}$
(500 nm particle size, 99.9\% purity, ABCR $\mathrm{GmbH}$ ) and $\mathrm{CaH}_{2}$ (99.99\%, Sigma Aldrich) in an Ar filled glove box in the molar proportion $1: 2.25$ (that is $\mathrm{BaTiO}_{3}: \mathrm{H}=1: 4.5$ ) and $1: 0.8$ (that is $\left.\mathrm{BaTiO}_{3}: \mathrm{H}=1: 1.6\right)$ for CA2 and CA3, respectively. Approximately $10 \mathrm{~g}$ of this mixture was pressed into pellets with a mass of about $1 \mathrm{~g}$. The pellets were placed inside sealed stainless steel ampules and heated in an evacuated silica jacket to a temperature of $600{ }^{\circ} \mathrm{C}$ for two days. A third sample, termed NAB, was prepared by mixing powders of $\mathrm{BaTiO}_{3}$ and $\mathrm{NaBH}_{4}(98 \%, \mathrm{ABCR}$ $\mathrm{GmbH})$ in an Ar filled glove box in the molar proportion $1: 0.45$ (that is $\mathrm{BaTiO}_{3}: \mathrm{H}=1: 1.8$ ). Pellets of this mixture were heated to a temperature of $600{ }^{\circ} \mathrm{C}$ for two days. The dark-blue coloured products were washed 3-4 times with a mixture of $0.1 \mathrm{M} \mathrm{NH}_{4} \mathrm{Cl}$ and methanol (CA2), 0.1 M acetic acid (CA3), or 0.1 M hydrochloric acid (NAB) in order to remove excess metal hydride and the byproducts $\mathrm{CaO}$ (CA2 and CA3) and $\mathrm{NaBO}_{2}$ (NAB). Finally, $\mathrm{CA} 2, \mathrm{CA} 3$ and $\mathrm{NAB}$ were dried under dynamic vacuum at $120^{\circ} \mathrm{C}$ for about one day. For the sample preparation of CA1, see ref. 4 .

\subsection{Powder X-ray diffraction}

PXRD patterns were collected at ambient temperature on a Panalytical X'Pert Alpha1 diffractometer operated with $\mathrm{Cu} \mathrm{K} \alpha 1$ radiation and in $\theta-2 \theta$ diffraction geometry. The powder samples were mounted on a $\mathrm{Si}$ wafer zero-background holder and diffraction patterns were measured in a $2 \theta$ range of $10-90^{\circ}$ with a step size of $0.013^{\circ}$. The Rietveld method as implemented in the FullProf program was used for structure and phase analysis.

\subsection{Thermal gravimetric analysis}

TGA experiments were carried out using a TA instruments Discovery system. The samples, each weighing approximately $15 \mathrm{mg}$, were heated in a platinum crucible from room temperature to $900{ }^{\circ} \mathrm{C}$ with a heating rate of $5{ }^{\circ} \mathrm{C} \mathrm{min}{ }^{-1}$. A dry air gas flow of $20 \mathrm{ml} \mathrm{min}{ }^{-1}$ was applied.

\subsection{Nuclear magnetic resonance spectroscopy}

The ${ }^{1} \mathrm{H}$ MAS NMR experiments were performed at a magnetic field of 9.4 $\mathrm{T}$ (400.12 $\mathrm{MHz}^{1} \mathrm{H}$ Larmor frequency) and a MAS rate of $30.00 \mathrm{kHz}$ on a Bruker Avance-III spectrometer equipped with a $2.5 \mathrm{~mm}$ MAS probehead. Acquisitions involved rotorsynchronized, double-adiabatic spin-echo sequence ${ }^{\mathbf{1 4 , 1 5}}$ with a $90^{\circ}$ excitation pulse of length $1.2 \mu$ s followed by two $50.0 \mu \mathrm{s}$ tanh/tan short high-power adiabatic pulses (SHAPs) ${ }^{16,17}$ with 5 $\mathrm{MHz}$ frequency sweep. All pulses operated at a nutation frequency of $208 \mathrm{kHz}$. For each sample 4096 signal transients with 5 s relaxation delays were accumulated. Shifts were referenced with respect to tetramethylsilane (TMS) at $0 \mathrm{ppm}$.

\subsection{Inelastic neutron scattering}

The INS experiments were performed on three instruments; on MERLIN $^{18,19}$ and TOSCA $^{20,21}$ at the ISIS Neutron and Muon Source (ISIS) at the Rutherford Appleton Laboratory, U.K., and on VISION ${ }^{22}$ at the Spallation Neutron Source (SNS) at the Oak Ridge National Laboratory, U.S. For the present work, the key difference between the spectrometers is that MERLIN enables 
both momentum $\left(Q, \AA^{-1}\right)$ and energy $(\omega, \mathrm{meV})$ transfer to be measured independently, whereas TOSCA and VISION follow a fixed trajectory in $(Q, \omega)$ space.

The experiment on MERLIN was performed on CA2 and CA3. Approximately $10 \mathrm{~g}$ of each sample was loaded into an aluminium sachet and the sachet into a thin-walled aluminium can. INS spectra were measured using three different incident energies of the neutrons: $E_{\mathrm{i}}=200 \mathrm{meV}(5 \mathrm{meV}, 400 \mathrm{~Hz}), 400$ $\mathrm{meV}(12 \mathrm{meV}, 550 \mathrm{~Hz})$ and $600 \mathrm{meV}(16 \mathrm{meV}, 600 \mathrm{~Hz})$, where the numbers within the parentheses refer to the resolution in energy and to the chopper frequency. Measurements were taken at $T=10,240$, and $280 \mathrm{~K}$ for CA2, and at $T=10 \mathrm{~K}$ for CA3. The time-of-flight data were first converted into INS maps, $S(Q, \omega)$, using the software MANTID, ${ }^{23}$ and the maps were subsequently analyzed using both the software MSLICE ${ }^{24}$ and DAVE. ${ }^{25}$ The very same samples (CA2 and CA3), using the same sample cells, were also measured on TOSCA at $T<10 \mathrm{~K}$. Additionally, $10 \mathrm{~g}$ of CA1, was also measured on TOSCA, with a flat sample cell, at $T<$ $10 \mathrm{~K}$.

The experiment on VISION was performed on $10 \mathrm{~g}$ of NAB, at $T<10 \mathrm{~K}$. The sample was thinly distributed inside an aluminum package that was subsequently rolled into a $54 \mathrm{~mm}$ long annuli with a diameter of $29 \mathrm{~mm}$. An aluminum can, which was vacuum sealed using aluminum foil, was used as a sample holder. The software DAVE was used for the analysis of $S(Q, \omega){ }^{25}$

2.5.1 Modeling of the INS spectra. INS relates to the inelastic scattering of neutrons from vibrating atoms. The INS intensity associated with the vibration of a specific atomic species depends on its neutron scattering cross section $\sigma$ and on both the energy and the momentum exchanged between the neutrons and the vibrating atoms. One should note that the total neutron scattering cross sections of $\mathrm{H}, \mathrm{Ba}, \mathrm{Ti}$, and $\mathrm{O}$ are 82.0, 3.38, 4.35, and 4.23 barns, respectively, ${ }^{26}$ and since the INS intensity depends on the amplitude of vibration, which is larger for light atoms, the measured spectra of $\mathrm{BaTiO}_{3-x} \mathrm{H}_{x}$ will be dominated by motions that involve displacements of the hydride ions. The following description is therefore limited to the vibrational modes involving the hydride ions, $\nu_{\mathrm{H}}$, which are predominantly localized in nature and essentially decoupled from the vibrational dynamics of the perovskite structure. ${ }^{13}$ The vibrational mode $\nu_{\mathrm{H}}$ can be described by its vibrational energy $\omega_{\nu}$ and the displacement vector $\boldsymbol{u}_{\mathrm{H}, \nu}$ along the direction of the vibration. The INS intensity associated with this mode can be expressed as $^{27,28}$

$$
S^{\prime}\left(\boldsymbol{Q}, \boldsymbol{\omega}_{\nu}\right)_{\mathrm{H}}^{(n)} \propto \frac{\left(\boldsymbol{Q} \cdot \boldsymbol{u}_{\mathrm{H}, \nu}\right)^{2 n}}{n !} \exp \left[-\left(\boldsymbol{Q} \cdot \sum_{\nu} \boldsymbol{u}_{\mathrm{H}, \nu}\right)^{2}\right],
$$

where $n=1$ are fundamental modes, $n=2$ their first overtones, $n=3$ their second overtones, and so forth. For a powder sample, which is the case here, the INS intensity is given by the average of eqn (1) taken with respect to all possible directions of $Q,{ }^{27,28}$ i.e.

$$
S\left(Q, \omega_{\nu}\right)=\frac{1}{4 \pi} \int S^{\prime}\left(\boldsymbol{Q}, \omega_{\nu}\right) \mathrm{d} \boldsymbol{Q}
$$

When the displacements $\boldsymbol{u}_{\mathrm{H}, \nu}$, associated with the local vibrational modes, are relatively close to each other, they can be approximated by

$$
\left|\boldsymbol{u}_{\mathrm{H}, \nu}\right|^{2} \approx \frac{1}{3} u_{\mathrm{H}, \mathrm{loc}}^{2} \quad \forall \nu .
$$

That is, the displacements are all similar to the same, average, value $u_{\mathrm{H}, \text { loc }}^{2} / 3$, where

$$
u_{\mathrm{H}, \mathrm{loc}}^{2}=\sum_{\nu=1}^{3}\left|\boldsymbol{u}_{\mathrm{H}, \nu}\right|^{2}
$$

is the MSD associated with the local vibrational motion, here denoted as local MSD. Using this approximation, which is referred to as the almost isotropic oscillator approximation, the INS intensity can be expressed as ${ }^{27,29}$

$$
S(Q, \omega)_{\mathrm{H}}^{(n)}=Q^{2 n} \gamma_{\mathrm{H}}^{(n)} \mathrm{e}^{-Q^{2} \alpha_{\mathrm{H}}^{(n)}},
$$

where, for fundamental modes,

$$
\begin{gathered}
\gamma_{\mathrm{H}, \nu}^{(1)} \propto u_{\mathrm{H}, \nu}^{2}, \quad \alpha_{\mathrm{H}, \nu}^{(1)}=\frac{1}{5}\left(u_{\mathrm{H}, \mathrm{tot}}^{2}+2 u_{\mathrm{H}, \nu}^{2}\right), \\
u_{\mathrm{H}, \text { tot }}^{2}=u_{\mathrm{H}, \mathrm{loc}}^{2}+u_{\mathrm{H}, \text { lattice }}^{2}
\end{gathered}
$$

and for first order overtones $\left(n=2, \omega=2 \omega_{\nu}\right)$ and binary combinations $\left(n=2, \omega=\omega_{\nu}+\omega_{\nu^{\prime}}\right)$ the coefficients $\alpha_{\mathrm{H}}$ and $\gamma_{\mathrm{H}}$ are given by

$$
\begin{gathered}
\gamma_{\mathrm{H}, v}^{(2)} \propto u_{\mathrm{H}, v}^{4}, \\
\alpha_{\mathrm{H}, v}^{(2)}=\frac{1}{7}\left(u_{\mathrm{H}, \mathrm{tot}}^{2}+4 u_{\mathrm{H}, v}^{2}\right), \\
\gamma_{\mathrm{H}, \mathrm{comb}}^{(2)} \propto u_{\mathrm{H}, \nu}^{2} u_{\mathrm{H}, v^{\prime}}^{2}, \\
\alpha_{\mathrm{H}, \mathrm{comb}}^{(2)}=\frac{1}{7}\left(u_{\mathrm{H}, \mathrm{tot}}^{2}+2 u_{\mathrm{H}, \nu}^{2}+2 u_{\mathrm{H}, v^{\prime}}^{2}\right)
\end{gathered}
$$

Here $u_{\mathrm{H}, \nu}^{2}$ stands for the scalar quantity $\left|\boldsymbol{u}_{\mathrm{H}, \nu}\right|^{2}, u_{\mathrm{H}, \text { tot }}^{2}$ is the total

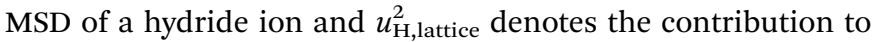
the total MSD from the motion of the hydride ion in phase with the collective perovskite lattice modes.

By differentiation of eqn (5) one can further derive that the maximum of $S(Q, \omega)_{\mathrm{H}}^{(n)}$ occurs when

$$
Q=Q_{\max }^{(n)}=\sqrt{n / \alpha_{\mathrm{H}}^{(n)}} .
$$

In the almost isotropic oscillator approximation, and assuming

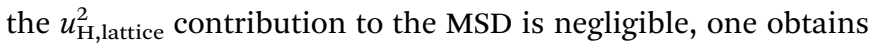

$$
\alpha_{\mathrm{H}, \nu}^{(1)} \approx \alpha_{\mathrm{H}, \nu}^{(2)} \approx \alpha_{\mathrm{H}, \mathrm{comb}}^{(2)} \simeq u_{\mathrm{H}, \mathrm{loc}}^{2} / 3,
$$

which leads to

$$
Q_{\max }^{(n)} \approx \sqrt{n} Q_{\max }^{(1)} .
$$

Because of the dependence of $Q_{\max }$ on $n$, the study of $Q$ profiles provides a means to distinguish between fundamental modes and higher-order transitions in the INS spectra. ${ }^{29,30}$ 
2.5.2 The case of hydride ions in $\mathrm{BaTiO}_{3}$. In $\mathrm{BaTiO}_{3-x} \mathrm{H}_{x}$ the hydride ions participate in the $\mathrm{Ti}-\mathrm{H}$ vibrational modes. One of these vibrational modes, $\omega_{\|}$, is parallel to the Ti-H-Ti bond direction, whereas the other two (degenerate) modes, $\omega_{\perp}$, are perpendicular to it. ${ }^{\mathbf{1 0 , 1 3}}$ Accordingly, the local MSD is written as

$$
u_{\mathrm{H}, \mathrm{loc}}^{2}=2 \cdot\left|\boldsymbol{u}_{\mathrm{H}, \perp}\right|^{2}+\left|\boldsymbol{u}_{\mathrm{H}, \|}\right|^{2},
$$

where $u_{\mathrm{H}, \|}^{2}$ and $u_{\mathrm{H}, \perp}^{2}$ are the respective MSD associated with the $\omega_{\|}$and $\omega_{\perp}$ vibrational modes. By combining eqn (6), (7) and (10) we obtain the following set of equations

$$
\begin{aligned}
\alpha_{\mathrm{H}, \|}^{(1)} & =\frac{1}{5}\left[u_{\mathrm{H}, \text { lattice }}^{2}+3 u_{\mathrm{H}, \|}^{2}+2 u_{\mathrm{H}, \perp}^{2}\right], \\
\alpha_{\mathrm{H}, \perp}^{(1)} & =\frac{1}{5}\left[u_{\mathrm{H}, \text { lattice }}^{2}+u_{\mathrm{H}, \|}^{2}+4 u_{\mathrm{H}, \perp}^{2}\right], \\
\alpha_{\mathrm{H}, \|}^{(2)} & =\frac{1}{7}\left[u_{\mathrm{H}, \text { lattice }}^{2}+5 u_{\mathrm{H}, \|}^{2}+2 u_{\mathrm{H}, \perp}^{2}\right], \\
\alpha_{\mathrm{H}, \perp}^{(2)} & =\frac{1}{7}\left[u_{\mathrm{H}, \text { lattice }}^{2}+u_{\mathrm{H}, \|}^{2}+6 u_{\mathrm{H}, \perp}^{2}\right] \\
\alpha_{\mathrm{H}, \text { comb }}^{(2)} & =\frac{1}{7}\left[u_{\mathrm{H}, \text { lattice }}^{2}+3 u_{\mathrm{H}, \|}^{2}+4 u_{\mathrm{H}, \perp}^{2}\right] .
\end{aligned}
$$

When the $\alpha$ coefficients are known, these equations can be used to derive the MSD associated with the hydride ions, i.e. $u_{\mathrm{H}, \perp}^{2}, u_{\mathrm{H}, \|}^{2}$ and $u_{\mathrm{H}, \text { lattice. }}^{2}$

\section{Computational details}

\subsection{Density functional theory calculations}

The DFT calculations were performed using the projector augmented wave (PAW) method $^{31,32}$ as implemented in the VASP $^{33,34}$ software together with the $\mathrm{PBE}^{35}$ exchange-correlation functional. A supercell containing 40 atoms constructed from a $2 \times 2 \times 2$ stacking of primitive unit cells was used in most calculations. We denote this the defect cell. The introduction of a hydride ion in the defect cell implies a defect concentration of $12.5 \%$. The same defect cell was used for the calculation of the combined defect of a hydride ion and an oxygen vacancy in various configurations. For the defect cell a Monkhorst-Pack $k$ point mesh of $6 \times 6 \times 6$ was used together with a plane wave cut-off energy of $500 \mathrm{eV}$. The calculations were converged to energies within $10^{-7} \mathrm{eV}$ for the electronic structure and to forces within $10^{-4} \mathrm{eV}^{-1}$ for the ionic relaxations.

\subsection{Vibrational density of states}

The vibrational frequencies and eigenmodes were obtained using the frozen phonon method in the harmonic approximation as implemented in Phonopy. ${ }^{36}$ Calculations of the force constants of the dynamical matrix were conducted in the defect cell equivalent to a supercell of $2 \times 2 \times 2$ primitive $\mathrm{BaTiO}_{3}$ unit cells. The convergence was checked with respect to supercell size by augmenting the defect cell dimension with a factor 2 in each direction to a $2 \times 2 \times 2$ defect cell of 320 atoms for the $\mathrm{H}_{\mathrm{O}}^{\times}$. This explicitly includes the zone-boundary points in the calculation. The difference for the phonon spectrum between the primitive and augmented defect cell was negligible and for the combined defect of both a hydride ion and an oxygen vacancy the smaller cell was used.

The projected vibrational density of states (PDOS) from a phonon calculation can be expressed as ${ }^{36}$

$$
g_{\alpha, i}(\omega)=\frac{1}{3 N} \sum_{\mathbf{q} s} \delta\left(\omega-\omega_{\mathbf{q} s}\right)\left|e_{\mathbf{q} s}^{\alpha, i}\right|^{2}
$$

where $\omega_{\mathbf{q} s}$ is the eigenfrequency of the dynamical matrix for band $s$ at the point $\boldsymbol{q}, e_{\mathbf{q} s}^{\alpha, i}$ is the corresponding eigenmode for atom $i$ in Cartesian direction $\alpha$ and $N$ is the number of atoms in the simulation cell. Eqn (12) allows a separation into the contribution from different atoms, $i$, and directions, $\alpha$. The total density of states (DOS), is obtained by the summation over all atoms and directions.

The MSD was calculated through

$$
\left\langle\left|u_{\alpha, i}(T)\right|^{2}\right\rangle=\int f_{i}(\omega, T) g_{\alpha, i}(\omega) \mathrm{d} \omega,
$$

where $g_{\alpha, i}(\omega)$ is the density of state defined in eqn (12) and

$$
f_{i}(\omega, T)=\frac{\hbar}{2 m_{i} \omega} \operatorname{coth}\left(\frac{\hbar \omega}{2 k_{\mathrm{B}} T}\right)
$$

is a temperature dependent weight factor, where $\hbar$ is Planck's constant, $k_{\mathrm{B}}$ Boltzmann's constant and $T$ the temperature in kelvin. In the low temperature limit, $k_{\mathrm{B}} T \ll \hbar \omega$, the weight factor $f_{i}(\omega, T) \rightarrow \hbar / 2 m_{i} \omega$. In the high temperature limit, $k_{\mathrm{B}} T \gg \hbar \omega$, the weight factor $f_{i}(\omega, T) \rightarrow k_{\mathrm{B}} T / m_{i} \omega^{2}$.

\section{Results}

\subsection{Average-structural and thermogravimetric analysis}

The average (long-range) structural and thermal properties of the four samples, CA1, CA2, CA3, and NAB were investigated with PXRD, TGA, and ${ }^{1} \mathrm{H}$ MAS NMR. The results of the average structural and thermal characterisation of CA1, CA2 and NAB were reported and discussed elsewhere. ${ }^{\mathbf{4 , 1 3}}$ The characterisation of $\mathrm{CA} 3$, together with that of CA2 and NAB, can be found in Fig. S1-S2 and Table S1 (PXRD), Fig. S3 (TGA), and Fig. S4 $\left({ }^{1} \mathrm{H}\right.$ MAS NMR) in the ESI $\dagger$ and a summary of the results for all the samples can be found in Table 1 . On the basis of the averagestructural analysis and TGA the composition of the metal hydride reduced $\mathrm{BaTiO}_{3}$ samples are reformulated as $\mathrm{BaTiO}_{3-x} \mathrm{H}_{y} \square_{x-y}$ with $x \approx 0.7$ and $y \approx 0.04$ for NAB, with $x \approx$ 0.35 and $y \approx 0.08$ for CA2, and with $x \approx 0.18$ and $y \approx 0.12$ for CA3 ( $c f$. Table 1). The heterogeneous nature of CA2 and CA3 (as suggested from the PXRD pattern) may be due to a phase segregation into a $\mathrm{O}$ vacancy rich and $\mathrm{H}$ rich phase. However, at this point this remains unclear.

\subsection{Inelastic neutron scattering}

4.2.1 Main features of the INS spectra. Fig. $1(\mathrm{a}-\mathrm{c})$ shows the INS spectra of CA1, CA2, CA3, and NAB at $T<10 \mathrm{~K}$, as measured on (a) MERLIN (CA2 and CA3), (b) TOSCA (CA1, CA2 and CA3), and (c) VISION (NAB), respectively. Apart from some differences in the relative intensity of the different contributions in the 
Table 1 Results of the characterization of CA1, CA2, CA3, and NAB ${ }^{a}$

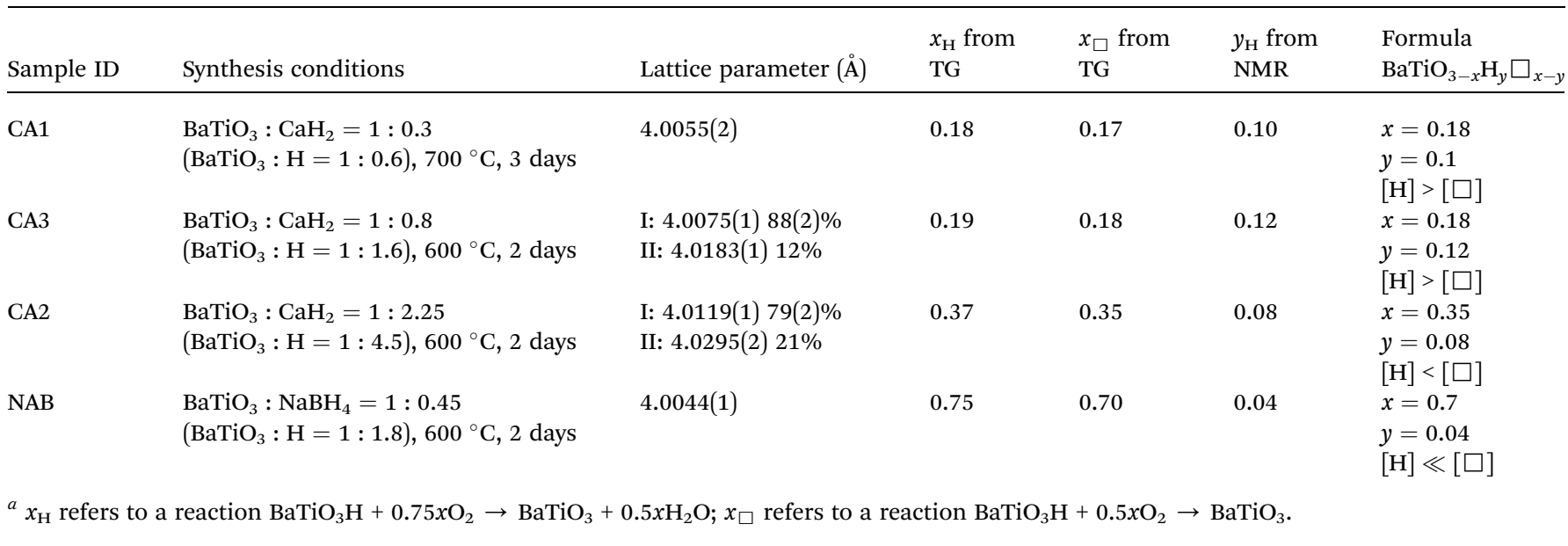

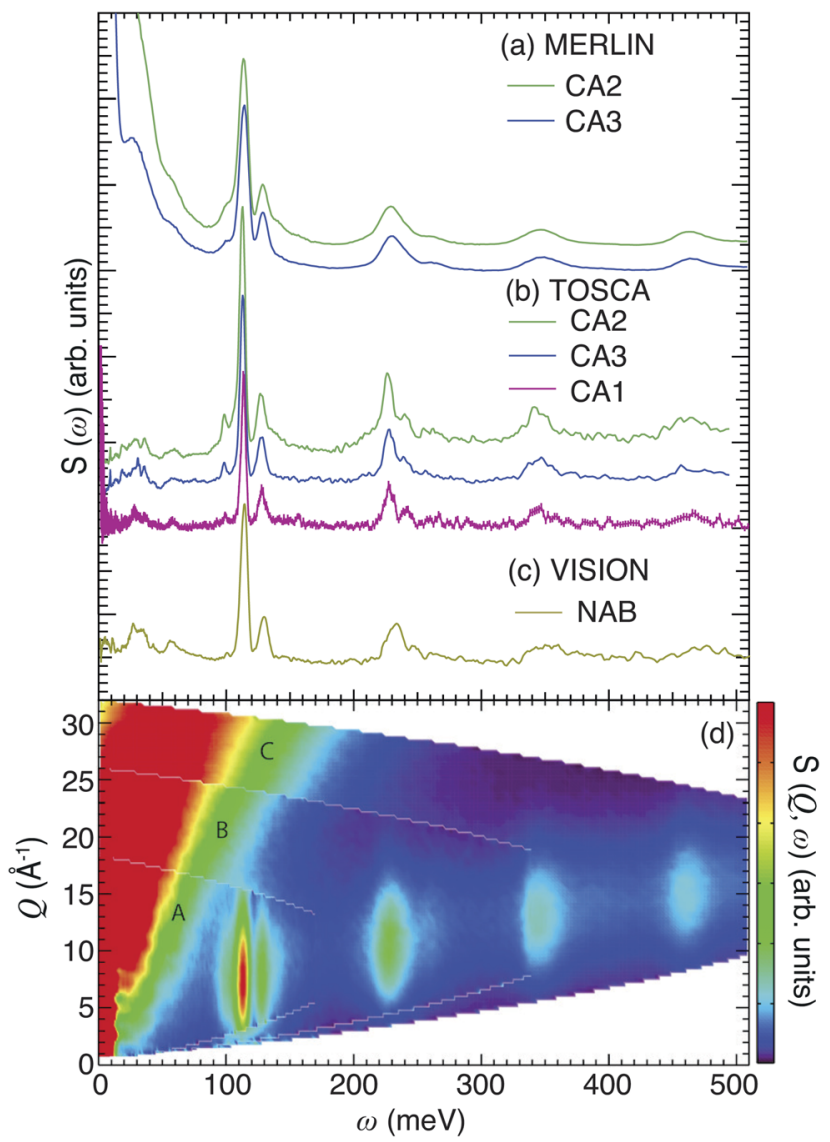

Fig. 1 INS spectra at $T<10 \mathrm{~K}$ of (a) CA2 and CA3 recorded on MERLIN and (b) CA2, CA3 and CA1 recorded on TOSCA, and of (c) NAB recorded on VISION. The different data sets have been vertically shifted for easier comparison. (d) $S(Q, \omega)$ maps of $C A 2$, as measured on MERLIN with three different energies of the incident neutrons, 200 $\mathrm{meV}(\mathrm{A}), 400 \mathrm{meV}(\mathrm{B})$ and $600 \mathrm{meV}$ (C). The INS intensity is shown according to the color-code bar. The INS spectra in (a) have been obtained by the integration of each $S(Q, \omega)$ map over the entire $Q$ range. spectra, the spectra for the different materials and measured on the three different instruments are overall similar and exhibit the same main features. Because the compositions and INS spectra of CA1 and CA3 are almost identical, $c f$. Table 1, we will in the forthcoming restrict our detailed analysis to CA3, for which the INS spectrum has a higher signal-to-noise ratio.

Above $90 \mathrm{meV}$ all spectra are characterized by a series of contributions, where the most intense is located at around 110 $\mathrm{meV}$, and the others are centered at approximately 230, 350 and $460 \mathrm{meV}$, respectively. In agreement with previous studies, these contributions relate to either fundamental $\mathrm{Ti}-\mathrm{H}$ vibrational modes or higher-order transitions of these modes, ${ }^{\mathbf{1 0 , 1 3}}$ whereas the bands at $<90 \mathrm{meV}$ are assigned to vibrational modes of the perovskite structure. ${ }^{13}$ This assignment is further supported by the fact that the INS spectrum and the computed vibrational density of states of $\mathrm{BaTiO}_{3}$ (Fig. S5-S7†) are essentially flat at $>90 \mathrm{meV}$. More specifically, the region between 90 and $150 \mathrm{meV}$ is characterized by two strong peaks at 100 and $130 \mathrm{meV}$, and a small side band at around $90 \mathrm{meV}$. These features are assigned to the localized vibrations of the hydrogen, ${ }^{\mathbf{1 0}, 13}$ whereas the bands at higher energies, $>150 \mathrm{meV}$, are assigned to higher-order transitions of these modes. This assignment is in agreement with the fact that these higherenergy bands are located at approximately two, three, and four times the frequency of the fundamental bands between 90 and $130 \mathrm{meV}$ as noted above, and is further supported by the relative intensities of the bands.

We also notice the lack of an $\mathrm{O}-\mathrm{H}$ stretch band, which is generally manifested as a broad continuum from approximately $440 \mathrm{meV}\left(\approx 3600 \mathrm{~cm}^{-1}\right)$ down to $370 \mathrm{meV}\left(\approx 3000 \mathrm{~cm}^{-1}\right)$ or even lower energy and which confirms that the hydrogen species in the oxyhydride of $\mathrm{BaTiO}_{3}$ are situated on vacant $\mathrm{O}$ sites rather than bound to oxygens of the perovskite structure. This is also confirmed by our ${ }^{1} \mathrm{H}$ NMR results (Fig. S4 $\dagger$ ), in which the negative shifts are an indication of $\mathrm{Ti}-\mathrm{H}$ species. No positive shifts, which would indicate the presence of $\mathrm{Ti}-\mathrm{O}-\mathrm{H}$ protic species, are observed. ${ }^{37}$

Fig. 1(d) shows, as an example, $S(Q, \omega)$ of CA2, as obtained from the MERLIN experiment with three different energies of 
the incident neutrons, $200 \mathrm{meV}$ (A), $400 \mathrm{meV}$ (B) and $600 \mathrm{meV}$ (C). Notice how the maximum intensity of the higher-energy bands generally occurs at larger $Q$ values as the transition energy increases [Fig. 1(d)], exactly as one expects for higherorder transitions. ${ }^{28}$ This further supports the assignment of the higher-frequency INS modes to higher-order transitions of $\mathrm{H}$ vibrational fundamentals. For a more quantitative analysis of the INS spectra, we have performed a peak fit analysis and band assignment, which is described below.
4.2.2 Peak fit analysis and band assignment. Fig. 2(a-l) shows the peak fit of the spectra of CA2 and CA3 at $T=10 \mathrm{~K}$, respectively, as recorded on MERLIN and TOSCA. Fig. 2(m-o) shows the peak fit of the spectra of NAB at $T=10 \mathrm{~K}$, as recorded on VISION. On the basis of the analysis of the energy and relative intensity of the different peaks, as described above, the spectra can be generally separated into fundamental $\mathrm{Ti}-\mathrm{H}$ modes (85-160 meV), second-order transitions (180-300 meV), and third-order transitions (300-410 meV), respectively.
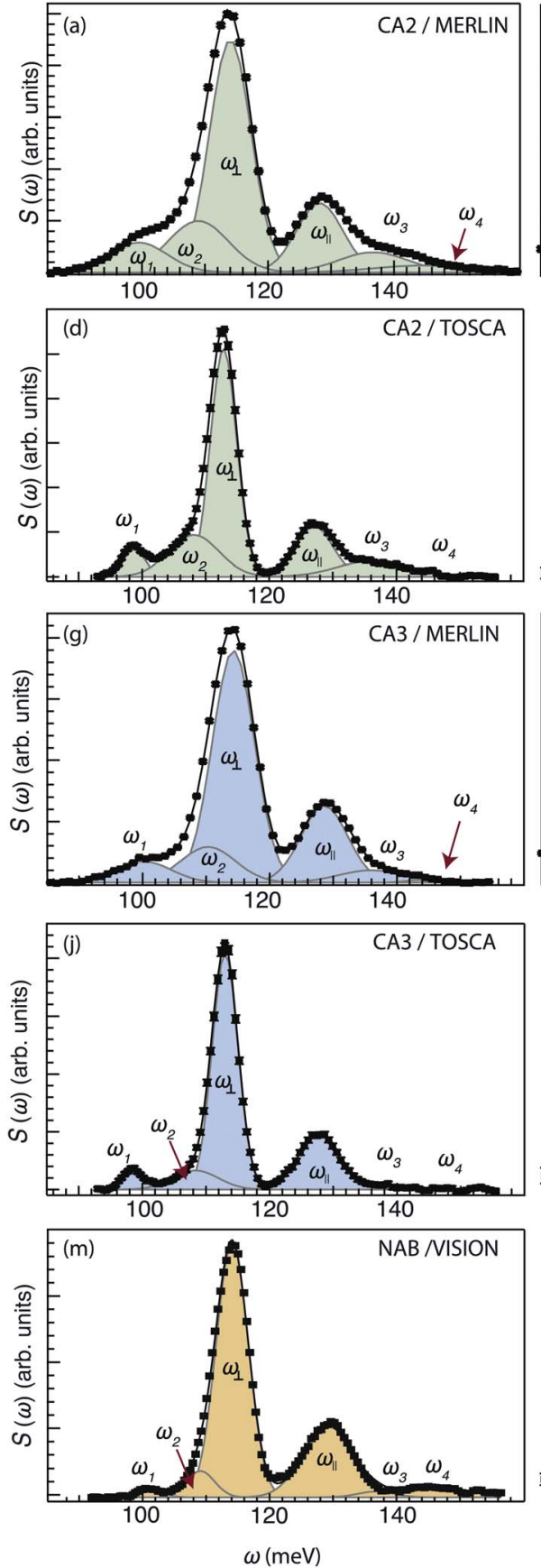
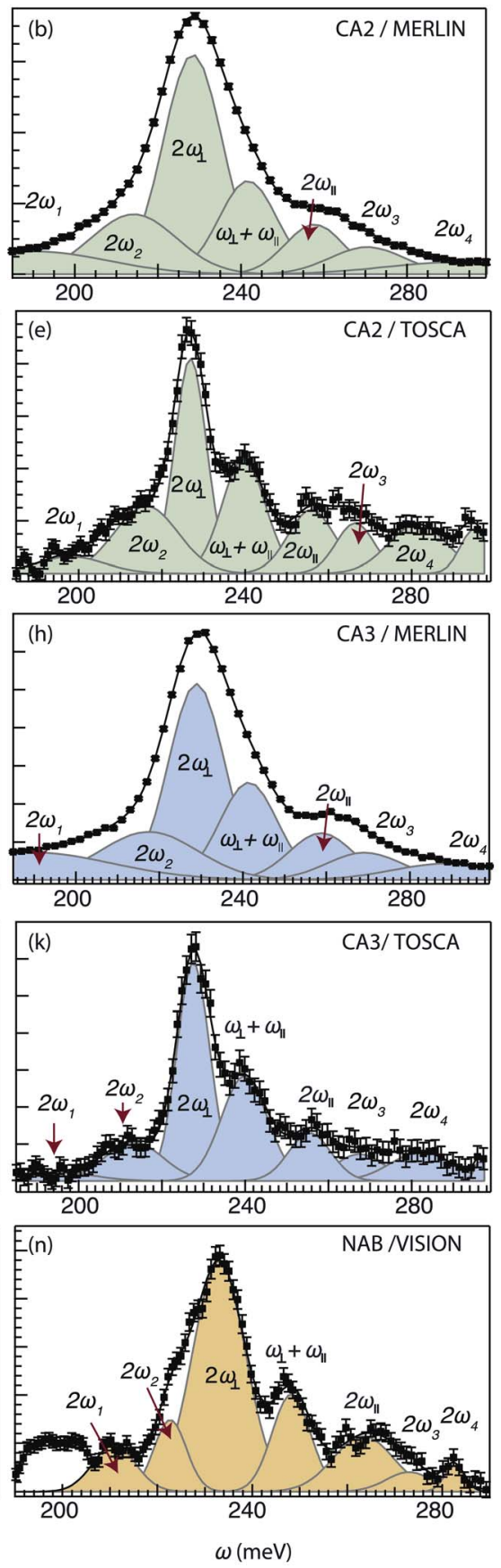
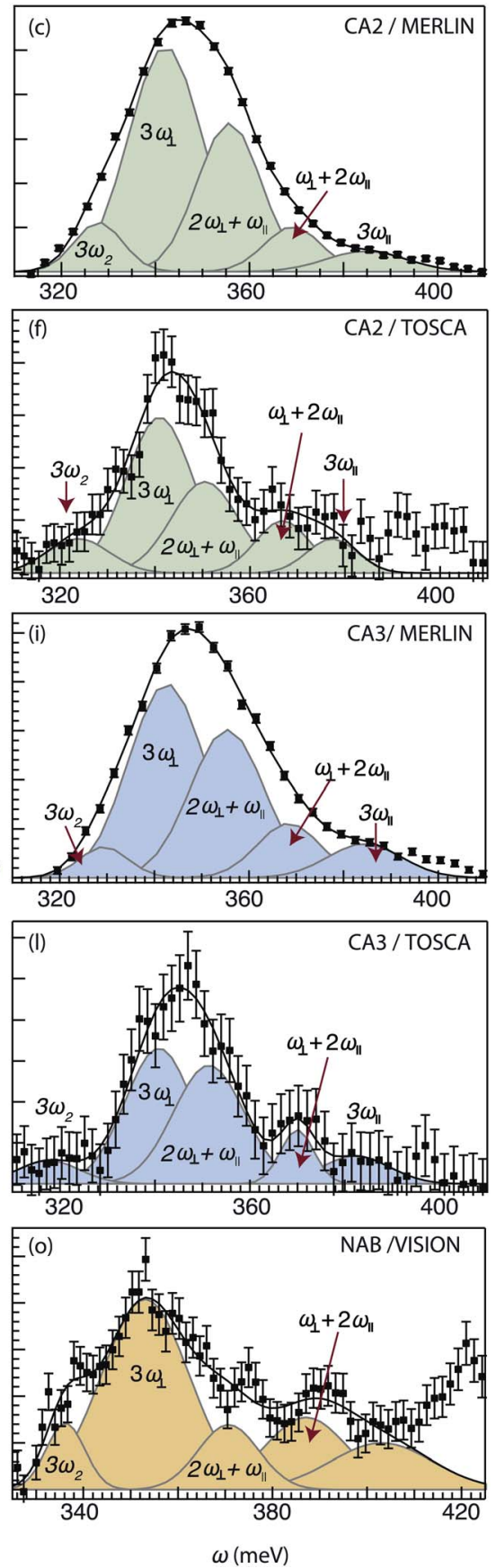

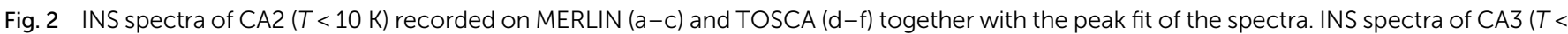

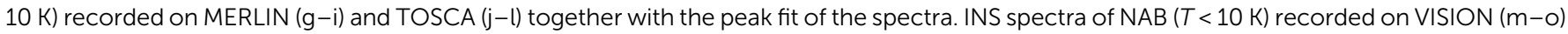
together with the peak fit of the spectra. 
For CA2, we find that the low-frequency region (85-160 meV) can be divided into six Gaussian components [Fig. 2(a and d)]. The two strongest bands, at $\omega \approx 114$ and $128 \mathrm{meV}$, are assigned to the fundamental $\mathrm{Ti}-\mathrm{H}$ vibrational modes $\omega_{\perp}$ and $\omega_{\|}$, respectively. Four weaker bands are located at $\approx 99\left(\omega_{1}\right), 110$ $\left(\omega_{2}\right), 137\left(\omega_{3}\right)$ and $145\left(\omega_{4}\right) \mathrm{meV}$, respectively. The presence of several bands related to $\mathrm{H}$ motions manifests the coexistence of several different $\mathrm{H}$ sites in the material.

The bands in the intermediate frequency range (180-300 $\mathrm{meV}$ ) can be adequately fit with seven Gaussians [Fig. 2(b and e)]. The three largest bands, at approximately 230, 240, and 255 $\mathrm{meV}$, are assigned, based on their frequencies and relative intensities with respect to each other and the bands at lower frequencies, as $2 \omega_{\|}, 2 \omega_{\perp}$ and $\omega_{\|}+\omega_{\perp}$, i.e. to overtones and combinations of the (main) fundamental contributions $\omega_{\|}$and $\omega_{\perp}$. In a similar manner, the remaining four bands are assigned as $2 \omega_{1}, 2 \omega_{2}, 2 \omega_{3}$ and $2 \omega_{4}$. Regarding possible combination modes related to $\omega_{1}-\omega_{4}$, we assume that they lie in the same frequency range as the overtones and combination of $\omega_{\|}$and $\omega_{\perp}$. Because they are less intense with respect to these latter ones, it is not possible to observe them.

The bands in the higher-frequency range (300-410 meV) can be adequately fit to five Gaussians, which are assigned as thirdorder transitions, i.e. $3 \omega_{2}, 3 \omega_{\|}, 3 \omega_{\perp}, 2 \omega_{\|}+\omega_{\perp}$ and $\omega_{\|}+2 \omega_{\perp}$ [Fig. 2(c and f)].

For the other two samples, CA3 and NAB [Fig. $2(\mathrm{~g}-\mathrm{l})$ and (mo)], we find that the peak fit analyses of the INS spectra yield comparable results to that of CA2. This is an important result that points toward no major differences in the local coordination environment of the hydride ions between the three samples. For CA2, we also recorded INS MERLIN spectra at elevated temperatures, $T=240$ and $T=280 \mathrm{~K}$ (Fig. S8 $\dagger$ ), which show no large spectral differences between the different temperatures. All the fit parameters are summarized in Table 2.

Note that the peak fit analysis for a specific sample as recorded on MERLIN and TOSCA both give essentially the same fit parameters, suggesting that the peak fit analysis is robust.

4.2.3 $Q$ profiles and mean-square displacements. The INS maps of CA2 and CA3, as measured on MERLIN, were subjected to an analysis of the $Q$-dependence of the different vibrational bands. For a specific spectral component $\omega_{\nu}, S(Q)$ was obtained by integration of $S(Q, \omega)$ over a narrow $\omega$ range centred on $\omega_{\nu}$. For the bands within the low-energy range, 85-160 meV, which are assigned as fundamental modes of the hydride ions, the $S(Q)$ curves were analysed using a modified version of eqn (5), i.e. $A+Q^{2 n} \gamma_{\mathrm{H}}^{(n)} \mathrm{e}^{-Q^{2} \alpha_{\mathrm{H}}^{(n)}}$, with $n=1$ and where the constant $A$ has been added in order to account for the possibility of a background in the spectra. For the bands assigned as second order transitions, in the medium-energy range, the $S(Q)$ curves were fitted using the same equation but with $n=2$. Fig. 3 shows $S(Q)$ for (a) $\omega_{\|}$and $2 \omega_{\|}$, and (b) $\omega_{\perp}$ and $2 \omega_{\perp}$ for CA2. As expected, the maximum of the INS signal occurs at larger $Q$ values as the order of the transition increases.

The fit parameters $\left(\gamma_{\mathrm{H}}\right.$ and $\left.\alpha_{\mathrm{H}}\right)$ are shown in Table 3. We observe that the $\gamma_{\mathrm{H}}$ and $\alpha_{\mathrm{H}}$ values are overall larger for CA2 compared to CA3. Since $\alpha_{\mathrm{H}}$ is proportional to $u_{\mathrm{H}, \text { int }}^{2}$, the hydride ions thus move with somewhat larger vibrational amplitude in
Table 2 Compilation of peak positions (in meV) of the Gaussianshaped components used to fit the spectra of CA2 and CA3 (as measured on TOSCA and MERLIN) and NAB (as measured on VISION). The peak positions have an uncertainty of the order of $1 \mathrm{meV}$

\begin{tabular}{|c|c|c|c|c|c|c|c|}
\hline \multirow[b]{2}{*}{ Sample } & \multicolumn{2}{|c|}{ TOSCA } & \multicolumn{4}{|c|}{ MERLIN } & \multirow{2}{*}{$\frac{\text { VISION }}{\text { NAB }}$} \\
\hline & CA3 & CA2 & CA3 & CA2 & CA2 & CA2 & \\
\hline$T(\mathrm{~K})$ & 10 & 10 & 9 & 9 & 240 & 280 & 10 \\
\hline$\omega_{1}$ & 98.4 & 98.5 & 100 & 99 & 100 & 100 & 101 \\
\hline$\omega_{2}$ & 108.4 & 108.1 & 110 & 109 & 109 & 109 & 109 \\
\hline$\omega_{\perp}$ & 112.9 & 112.8 & 114 & 114 & 114 & 114 & 114 \\
\hline$\omega_{\|}$ & 127.7 & 127.1 & 129 & 128 & 127 & 127 & 129 \\
\hline$\omega_{3}$ & 137.7 & 135.7 & 136 & 137 & 136 & 136 & 137 \\
\hline$\omega_{4}$ & 147.0 & 145.4 & 144 & 144 & 142 & 143 & 147 \\
\hline $2 \omega_{1}$ & 195.5 & 198.1 & 189 & 190 & 19 & 90 & 211 \\
\hline $2 \omega_{2}$ & 213.2 & 215.4 & 218 & 214 & 214 & 214 & 223 \\
\hline $2 \omega_{\perp}$ & 227.5 & 226.9 & 229 & 228 & 228 & 228 & 233 \\
\hline$\omega_{\perp}+\omega_{\perp}$ & 239.8 & 239.6 & 241 & 242 & 242 & 242 & 248 \\
\hline $2 \omega_{\|}$ & 255.5 & 255.9 & 259 & 258 & 258 & 257 & 263 \\
\hline $2 \omega_{3}$ & 267.4 & 266.4 & 269 & 270 & 271 & 271 & 273 \\
\hline $2 \omega_{4}$ & 282.3 & 281.4 & 289 & 292 & 294 & 296 & 283 \\
\hline $3 \omega_{2}$ & 317.6 & 323.8 & 329 & 328 & & & 336 \\
\hline $3 \omega_{\perp}$ & 340.8 & 340.9 & 343 & 342 & & & 353 \\
\hline $2 \omega_{\perp}+\omega_{\perp}$ & 351.4 & 350.5 & 355 & 356 & & & 371 \\
\hline$\omega_{\perp}+2 \omega_{\perp}$ & 370.0 & 366.9 & 368 & 369 & & & 387 \\
\hline $3 \omega_{\|}$ & 382.2 & 377.3 & 384 & 385 & & & 403 \\
\hline
\end{tabular}

CA2. As for the temperature dependence (investigated for CA2 only), $\gamma_{\mathrm{H}}$ decreases as a function of increasing temperature, whereas for $\alpha_{\mathrm{H}}$ we observe no apparent temperature dependence.

We also consider the effect of the order of the transition $(n)$ on the peak fitted parameters. As for the $\gamma_{\mathbf{H}}$ values, these decrease as a function of increasing $n$, which reflects the fact that higher-order transitions occur less likely than fundamental transitions. We also observe that $\gamma_{\mathrm{H}, \perp}^{(n)}>\gamma_{\mathrm{H}, \|}^{(n)}$, which reflects the fact that there are two perpendicular components and one parallel component of the hydride ion motion (Fig. 4). As for the $\alpha_{\mathbf{H}}$ values, these are virtually unaffected by the order of the transition, which is as expected for the overtone progression of an almost isotropic oscillator.
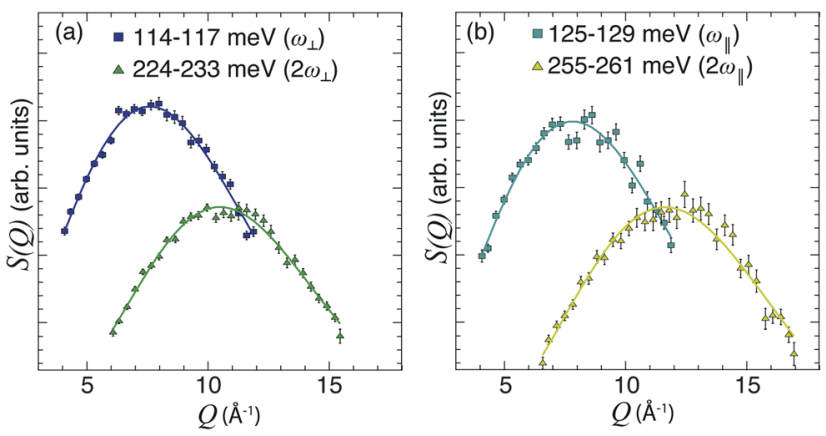

Fig. 3 (a and b) $S(Q)$ curves for $C A 2$ at $T=9 \mathrm{~K}$, as obtained after integration of $S(Q, \omega)$ over the indicated $\omega$ ranges (see legend), together with fits according to eqn (5) with $n=1$ and 2, respectively. 
Table 3 (a and b) Compilation of $\gamma_{\mathrm{H}}$ and $\alpha_{\mathrm{H}}$ for CA3 at $T=9 \mathrm{~K}$ and CA2 at $T=9,240$ and $280 \mathrm{~K}$. (c) Compilation of the MSDs of the hydrogen, as calculated from $\alpha_{\mathrm{H}}$. The numbers between parenthesis are the uncertainty in the last digit

\begin{tabular}{|c|c|c|c|c|}
\hline Sample & CA3 & CA2 & CA2 & CA2 \\
\hline$T(\mathrm{~K})$ & 9 & 9 & 240 & 280 \\
\hline \multicolumn{5}{|c|}{ (a) $\gamma_{H}$ (arb units) } \\
\hline$\omega_{1}$ & $1.46(7)$ & $1.9(1)$ & $1.7(1)$ & $1.5(1)$ \\
\hline$\omega_{2}$ & $2.27(9)$ & $3.6(1)$ & $4.0(2)$ & $4.1(2)$ \\
\hline$\omega_{\perp}$ & $11.0(2)$ & $11.5(2)$ & $10.5(3)$ & $9.94(30)$ \\
\hline$\omega_{\|}$ & $3.7(1)$ & $3.9(2)$ & $3.3(2)$ & $3.3(1)$ \\
\hline$\omega_{3}$ & $1.36(5)$ & $1.6(1)$ & $1.8(1)$ & $1.7(1)$ \\
\hline$\omega_{4}$ & $0.91(6)$ & $1.00(5)$ & $1.14(6)$ & $1.18(5)$ \\
\hline $2 \omega_{1}$ & $0.0086(3)$ & $0.0090(5)$ & $0.0091(4)$ & $0.0091(3)$ \\
\hline $2 \omega_{2}$ & $0.0144(4)$ & $0.0200(6)$ & $0.0228(5)$ & $0.0222(5)$ \\
\hline $2 \omega_{\perp}$ & $0.0477(7)$ & $0.052(1)$ & $0.0467(7)$ & $0.0454(6)$ \\
\hline$\omega_{\perp}+\omega_{\|}$ & $0.0212(5)$ & $0.0243(8)$ & $0.0249(6)$ & $0.0243(6)$ \\
\hline $2 \omega_{\|}$ & $0.0130(3)$ & $0.0142(5)$ & $0.0145(3)$ & $0.0147(3)$ \\
\hline $2 \omega_{3}$ & $0.00858(2)$ & $0.0100(4)$ & $0.0103(3)$ & $0.0098(3)$ \\
\hline $2 \omega_{4}$ & $0.0064(2)$ & $0.068(4)$ & $0.0077(2)$ & $0.0078(2)$ \\
\hline \multicolumn{5}{|c|}{ (b) $\alpha_{\mathrm{H}}\left(10^{-2} \stackrel{\circ}{ }^{2}\right)$} \\
\hline$\omega_{1}$ & $1.40(2)$ & $1.58(2)$ & $1.44(3)$ & $1.64(2)$ \\
\hline$\omega_{2}$ & $1.54(2)$ & $1.75(2)$ & $1.73(3)$ & $1.70(2)$ \\
\hline$\omega_{\perp}$ & $1.78(1)$ & $1.74(1)$ & $1.82(1)$ & $1.86(1)$ \\
\hline$\omega_{\|}$ & $1.59(1)$ & $1.64(1)$ & $1.59(2)$ & $1.56(2)$ \\
\hline$\omega_{3}$ & $1.19(1)$ & $1.31(2)$ & $1.30(2)$ & $1.29(1)$ \\
\hline$\omega_{4}$ & $0.99(2)$ & $1.09(1)$ & $1.12(2)$ & $1.10(1)$ \\
\hline $2 \omega_{1}$ & $1.73(2)$ & $1.76(2)$ & $1.71(2)$ & $1.70(1)$ \\
\hline $2 \omega_{2}$ & $1.75(1)$ & $1.83(2)$ & $1.83(1)$ & $1.85(1)$ \\
\hline $2 \omega_{\perp}$ & $1.79(1)$ & $1.82(1)$ & $1.82(1)$ & $1.83(1)$ \\
\hline$\omega_{\perp}+\omega_{\|}$ & $1.60(1)$ & $1.66(1)$ & $1.67(1)$ & $1.67(1)$ \\
\hline $2 \omega_{\|}$ & $1.44(1)$ & $1.48(1)$ & $1.49(1)$ & $1.49(1)$ \\
\hline $2 \omega_{3}$ & $1.31(1)$ & $1.38(1)$ & $1.38(1)$ & $1.36(1)$ \\
\hline $2 \omega_{4}$ & $1.23(1)$ & $1.28(1)$ & $1.26(1)$ & $1.28(1)$ \\
\hline \multicolumn{5}{|c|}{ (c) $\operatorname{MSD}\left(10^{-2} \AA^{2}\right)$} \\
\hline$u_{\mathrm{H}, \perp}^{2}$ & $1.72(6)$ & $1.8(2)$ & $1.82(2)$ & $1.87(7)$ \\
\hline$u_{\mathrm{H}, \|}^{2, \pm}$ & $1.14(6)$ & $1.3(2)$ & $1.25(2)$ & $1.23(7)$ \\
\hline$u_{\mathrm{H}, \text { lattice }}^{2}$ & $1.0(3)$ & $0.4(9)$ & $0.61(1)$ & $0.5(4)$ \\
\hline$u_{\mathrm{H}, \mathrm{loc}}^{2}$ & $4.58(13)$ & $4.9(4)$ & $4.89(4)$ & $5.0(2)$ \\
\hline
\end{tabular}

By using the $\alpha_{\mathrm{H}}$ values from Table 3 as input in expressions for the $\alpha_{\mathrm{H}}$ coefficients [eqn (11)] we have derived the corresponding MSD of the hydride ions, $u_{\mathrm{H}, \perp}^{2}, u_{\mathrm{H}, \|}^{2}, u_{\mathrm{H}, \mathrm{loc}}^{2}=2 \cdot u_{\mathrm{H}, \perp}^{2}+$ $u_{\mathrm{H}, \|}^{2}$ and $u_{\mathrm{H}, \text { lattice }}^{2}$ (Table 3). In agreement with the $\alpha_{\mathrm{H}}$ values, the hydride ions in the CA2 sample are manifested by larger local MSDs than the ones for CA3. Additionally, we remark that $u_{\mathrm{H}, \text { loc }}^{2}>u_{\mathrm{H} \text {,lattice, }}^{2}$ as one would expect for localized vibrational modes.

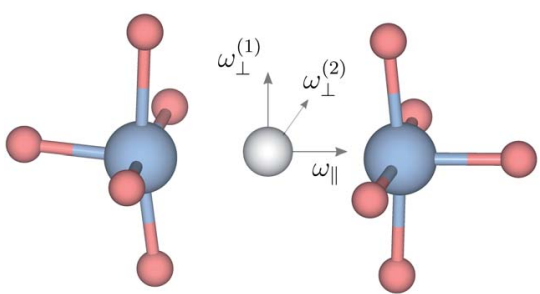

Fig. 4 Sketch indicating the direction of the $\omega_{\perp}$ and $\omega_{\|} \mathrm{Ti}-\mathrm{H}$ vibrational motions in metal hydride reduced $\mathrm{BaTiO}_{3}$.

\subsection{Density functional theory calculations}

DFT calculations were used to compute the hydride ion vibrational frequencies and MSD for three different configurations, containing a hydride ion and an oxygen vacancy as a first, second and third nearest neighbour, respectively. These configurations are denoted as $\mathrm{H}_{\mathrm{O}} v_{\mathrm{O}}^{\times(\dagger)}$ with $\dagger=1$, 2, 3. All configurations are neutral with three electrons in the conduction band. Fig. 5 illustrates a $\mathrm{H}_{\mathrm{O}} v_{\mathrm{O}}^{\times}$supercell where the numbers in the figure indicate the possible oxygen vacancy positions. With the oxygen vacancy as a second nearest neighbour there are two crystallographically non-equivalent positions (denoted as 2 and $2^{\prime}$ ). The configuration we consider is where the oxygen vacancy is located in the direction perpendicular to the $\mathrm{Ti}-\mathrm{H}$ bond, i.e. $\dagger=2$. The respective hydride ion frequencies, relative ground state energies $\Delta E$, and the multiplicity $g$ for the three different configurations are presented in Table 4 .

The energetically most favourable position is the one in which the oxygen is the second nearest neighbour, $\mathrm{H}_{\mathrm{O}} \mathrm{v}_{\mathrm{O}}^{\times(2)}$. The energy of the first nearest neighbour configuration $\mathrm{H}_{\mathrm{O}} \mathrm{V}_{\mathrm{O}}^{\times(1)}$ is about $0.1 \mathrm{eV}$ higher. This is not surprising since a repulsion of the two positively charged defects is to be expected. Also with the oxygen vacancy as third nearest neighbour the relative ground state energy is about $0.1 \mathrm{eV}$ higher. The second nearest neighbour position $2^{\prime}$ and the other possible configurations containing the combination of a vacancy and a hydride ion in this supercell size exhibit all relative ground state energies $>0.15 \mathrm{eV}$ and are not discussed further.

With respect to the probability for the occupancy of a particular configuration $\dagger$, this can be expressed as

$$
P^{(\dagger)} \propto g^{(\dagger)} \exp \left[-E^{(\dagger)} / k_{\mathrm{B}} T\right],
$$

where $g^{(\dagger)}$ is the multiplicity of configuration $\dagger$ with energy $E^{(\dagger)}$. At $600{ }^{\circ} \mathrm{C}$, where the material is kept for two days in the synthesis, the relative probabilities become $P_{1} / P_{2}=0.54$ and $P_{3} /$ $P_{2}=1.06$. Upon cooling, these relative probabilities decrease

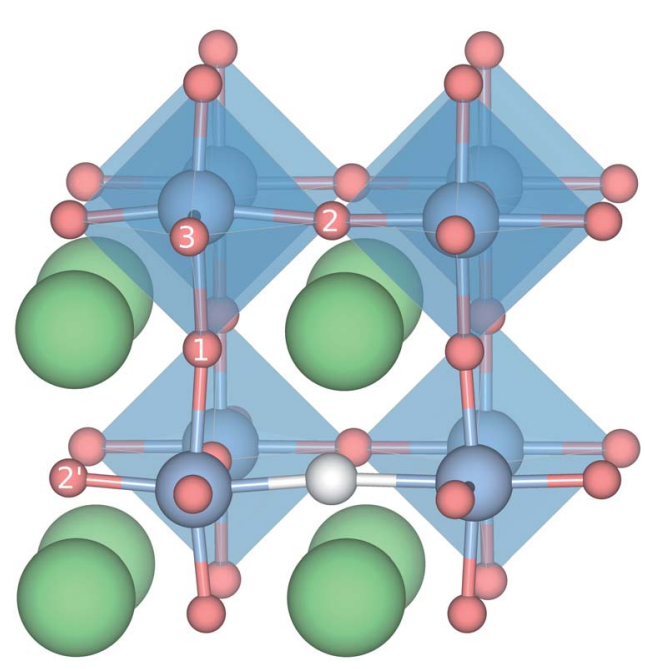

Fig. 5 Illustration of the different configurations containing both a $\mathrm{H}_{\odot}$ and $a v_{O}$ defect. The different positions for the oxygen vacancy are indicated by the numbers 1,2 , and 3 . 
Table 4 Vibrational frequencies (in $\mathrm{meV}$ ) for the local hydride ion vibrational modes in the three different defect configurations, and for a lattice without oxygen vacancies for the bandstate (band) and the polaron (pol) state, respectively. Values within parentheses refer to frequencies determined in ref. 13 with the use of the HSE functional. $\Delta E$ (in $\mathrm{meV}$ ) is the relative ground state energies and $g$ the multiplicity of the different configurations

\begin{tabular}{llllll}
\hline & $\mathrm{H}_{\mathrm{O}} \mathrm{v}_{\mathrm{O}}^{\times(1)}$ & $\mathrm{H}_{\mathrm{O}} \mathrm{V}_{\mathrm{O}}^{\times(2)}$ & $\mathrm{H}_{\mathrm{O}} \mathrm{v}_{\mathrm{O}}^{\times(3)}$ & $\mathrm{H}_{\mathrm{O}}^{\times}(\mathrm{band})^{13}$ & $\mathrm{H}_{\mathrm{O}}^{\times}(\mathrm{pol})^{13}$ \\
\hline$\omega_{\|}$ & 124 & 127 & 125 & $124(128)$ & $141(144)$ \\
$\omega_{\perp}^{(1)}$ & 108 & 105 & 106 & $106(111)$ & $104(111)$ \\
$\omega_{\perp}^{(2)}$ & 93 & 104 & 105 & - & $101(108)$ \\
$\Delta E$ & 99 & 0 & 100 & - & - \\
$g$ & 8 & 4 & 16 & - & -
\end{tabular}

and at $100{ }^{\circ} \mathrm{C}$ they are $P_{1} / P_{2}=0.09$ and $P_{3} / P_{2}=0.18$. Oxygen vacancy diffusion becomes slower upon cooling. Eventually configurations will be frozen-in in the cooling process and we expect that also at very low temperatures the configurations $\mathrm{H}_{\mathrm{O}} \mathrm{v}_{\mathrm{O}}^{\times(1)}$ and $\mathrm{H}_{\mathrm{O}} \mathrm{v}_{\mathrm{O}}^{\times(3)}$ are present.

4.3.1 Vibrational density of states. Fig. 6 shows the PDOS for the hydride ion in the energetically most favourable configuration, $\mathrm{H}_{\mathrm{O}} \mathrm{v}_{\mathrm{O}}^{\times(2)}$. The different colours represent different Cartesian directions and the total DOS for the hydride ion, which is the sum over the three Cartesian directions, is marked with grey color. The two intense peaks at $>90 \mathrm{meV}$ are assigned to hydrogen motions in directions parallel $\left(\omega_{\|}\right)$and perpendicular $\left(\omega_{\perp}\right)$ to the $\mathrm{Ti}-\mathrm{H}$ bond direction, whereas there are only small contributions from hydrogen motions at lower energies of the spectrum. Specifically, these low-energy hydrogen motions are associated with the participation of hydrogen in the collective lattice modes of the perovskite framework. The intensity of these modes is very weak and has been amplified by a factor of 100 in Fig. 6 for improved visibility.

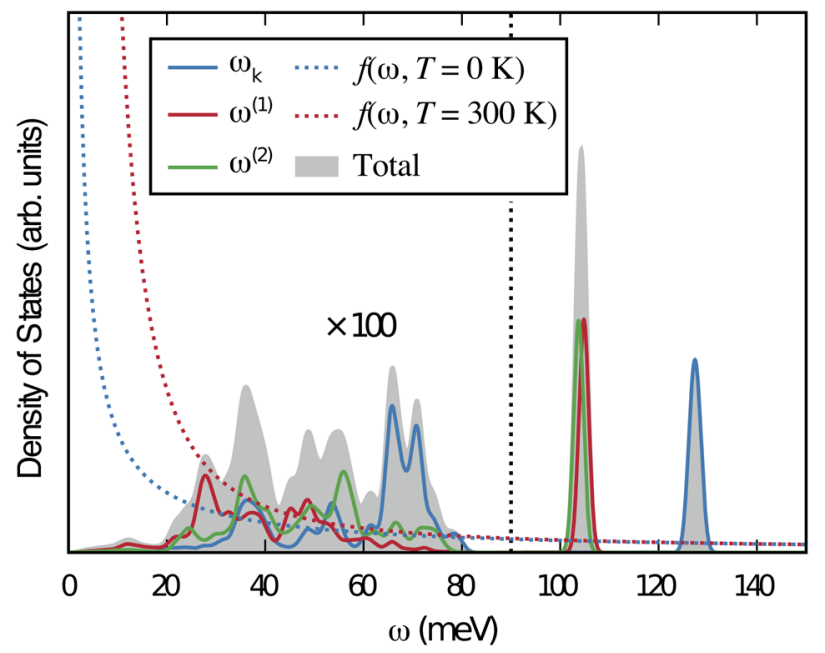

Fig. 6 PDOS for the hydride ion in the $\mathrm{H}_{\mathrm{OV}} \mathrm{O}^{\times(2)}$ configuration along the 3 Cartesian directions (colour) together with the total DOS (gray). The intensity for frequencies below $90 \mathrm{meV}$ are amplified by a factor 100 . The weight factor $f(\omega, T)$ [see eqn (14)] is also shown for two different temperatures.
Table 4 shows the vibrational frequencies for the localized hydrogen modes in the three different defect configurations, containing a hydride ion and an oxygen vacancy as first, second and third nearest neighbour, respectively. Included in the table are also the hydride vibrational frequencies in a structure without oxygen vacancies, for the case when the extra electron is delocalized over all $\mathrm{Ti}^{4+}$ ions (bandstate) and where the excess electron is localized on a titanium atom nearest neighbour to the hydride ion (polaron state), which have been computed previously. ${ }^{13}$

We remark that the results presented here are computed using a $2 \times 2 \times 2$ supercell, corresponding to the chemical composition BaTiO $_{2.75} \mathrm{H}_{0.125} \square_{0.125}$. To test for possible concentration dependencies of the (calculated) vibrational frequencies, we have also computed the frequencies in a $3 \times 3$ $\times 3$ supercell, corresponding to $\mathrm{BaTiO}_{2.926} \mathrm{H}_{0.037} \square_{0.037}$, for both cases of a structure without oxygen vacancies (bandstate) and a structure with second nearest neighbouring vacancies. The corresponding results are summarized in Fig. S6. $\dagger$ The frequencies for 1,2 and $3\left(\mathrm{H}_{\mathrm{O}} \mathrm{V}_{\mathrm{O}}^{\times}\right)$-defects, in a $3 \times 3 \times 3$ supercell have also been computed, corresponding to the compositions $\mathrm{BaTiO}_{2.926} \mathrm{H}_{0.037} \square_{0.037}, \mathrm{BaTiO}_{2.926} \mathrm{H}_{0.074} \square_{0.074}$, and $\mathrm{BaTiO}_{2.926} \mathrm{H}_{0.111} \square_{0.111}$, respectively. The oxygen vacancy is in all cases located at a second nearest neighboring oxygen site, the most stable configuration. The corresponding results are summarized in Fig. S7. $\dagger$ In all cases we find very sharp vibrational peaks which clearly shows the localized nature of the hydride ion vibrational motion. The very sharp nature of the peaks is a direct evidence for the localized nature. The local surrounding for a hydride ion in the different numerically investigated cases differ slightly which results in small shifts of the corresponding frequencies. In the INS measurement a weighted average is then obtained and it shows up as a broadening of the measured peak.

From the results in Table 4 we can observe that the hydrogen vibrational frequencies for the $\mathrm{H}_{\mathrm{O}} \mathrm{v}_{\mathrm{O}}^{\times(2)}$ configuration are very similar to both the $\mathrm{H}_{\mathrm{O}} \mathrm{V}_{\mathrm{O}}^{\times(3)}$ configuration and to the bandstate configuration. All configurations, except the bandstate configuration, break the degeneracy of the perpendicular mode, but only very weakly. The exception is the $\mathrm{H}_{\mathrm{O}} \mathrm{V}_{\mathrm{O}}^{\times(1)}$ configuration, where there is a significant downshift of about $12 \mathrm{meV}$ of one of the perpendicular modes, the one in the plane containing the vacancy. Based on this we expect the vibrational spectrum to be similar regardless of the vacancy concentration, and if the $\mathrm{H}_{\mathrm{O}} \mathrm{v}_{\mathrm{O}}^{\times(1)}$ configuration is present, a peak at lower frequencies should be present. Furthermore, we observe that the formation of a polaron shifts the frequency of the parallel mode, by about $15 \mathrm{meV}$, while the perpendicular modes remain essentially unchanged. This feature may thus be used as a means to determine the respective contribution of the bandstate and polaron state configurations in the material, in the case both states co-exist.

4.3.2 Mean square displacement. Table 5 shows the MSD of the localized $\left(u_{\text {loc }}^{2}\right)$ and lattice $\left(u_{\text {lattice }}^{2}\right)$ modes of the hydride ion, respectively, as derived from the computer simulations. Specifically, the respective MSD values have been computed by separating contributions in eqn (13) into two terms, according 
Table 5 MSD for the hydride ion in configuration $\mathrm{H}_{O} \mathrm{v}_{\mathrm{O}}^{\times(2)}$, divided into local and lattice contributions, at $T=0$ and $300 \mathrm{~K}$

\begin{tabular}{|c|c|c|c|c|c|c|c|c|}
\hline \multirow[b]{2}{*}{$T(\mathrm{~K})$} & \multicolumn{4}{|c|}{$u_{\text {lattice }}^{2}(\omega<90 \mathrm{meV})$ in $\times 10^{-2} \AA^{2}$} & \multicolumn{4}{|c|}{$\begin{array}{l}u_{\text {loc }}^{2} \\
(\omega>90 \mathrm{meV}) \text { in } \times 10^{-2} \AA^{2}\end{array}$} \\
\hline & $u_{\text {lattice }}^{2}$ & $u_{\text {lattice }}^{2} \stackrel{(1)}{\perp}$ & $u_{\text {lattice }}^{2} \stackrel{(2)}{\perp}$ & $u_{\text {lattice,tot }}^{2}$ & $u_{\mathrm{loc} \|}^{2}$ & $u_{\mathrm{loc} \perp}^{2}$ & $u_{\mathrm{loc} \perp}^{2}$ & $u_{\text {loc }}^{2}$ \\
\hline & 0.14 & 0.16 & 0.15 & 0.44 & 1.57 & 1.93 & 1.94 & 5.44 \\
\hline 00 & 0.28 & 0.42 & 0.31 & 1.01 & 1.60 & 2.00 & 2.01 & 5.60 \\
\hline
\end{tabular}

to whether the mode frequency is above or below $90 \mathrm{meV}$. This approach is fully justified because the hydride ion vibrations are localized and fully distinct from the lattice modes, as discussed in the previous sections. The $u_{\text {loc }}^{2}$ values are indeed found to be an order of magnitude larger than the $u_{\text {lattice values, which is }}^{2}$ consistent with the fact that the intensity of the contribution of the hydride ion in the region of the lattice vibrations, i.e. below $90 \mathrm{meV}$, is very weak (see Fig. 6).

Included in Fig. 6 is also the weight factor $f(\omega, T)$ [see eqn (14)] at two different temperatures, $T=0 \mathrm{~K}$ and $300 \mathrm{~K}$, together with the vibrational DOS. From the weight factor we observe that low frequency vibrations give a larger contribution to the MSD compared with high frequency vibrations and their contribution also increases more rapidly when the temperature is increased. For example, when the temperature is increased from $T=0 \mathrm{~K}$ to $300 \mathrm{~K}$, a mode with frequency $\hbar \omega=22 \mathrm{meV}$ gets an increased weight by a factor of 2.5 , while a mode with frequency $\hbar \omega=120$ meV only gets a factor of 1.02 increase. This implies that $u_{\text {ext }}^{2}$ has a significant temperature dependence compared to $u_{\text {int }}^{2}(c f$. Table 5). At low frequencies, $\hbar \omega<30 \mathrm{meV}$, the mode $\omega_{\perp}^{(1)}$ has the largest contribution to the vibrational DOS and hence $u_{\text {ext }}^{2}{ }_{\perp}^{(1)}$ has the largest temperature dependence ( $c f$. Table 5).

\section{Discussion}

In agreement with our previous work on metal hydride reduced $\mathrm{BaTiO}_{3}$ materials, ${ }^{\mathbf{1 3}, 37}$ the spectra show no indications of $\mathrm{O}-\mathrm{H}$ groups or other interstitial hydrogen species present in any of the materials. This establishes that the hydrogen species are present as hydride ions located on vacant oxygen sites of the perovskite structure, and not as protonic species, virtually independent of the level of hydride ion and oxygen vacancy concentrations as investigated here. In this context, we have followed the approach of Liu et al. ${ }^{7,8}$ and investigated the possibility, in $\mathrm{BaTiO}_{3}$, of what has been referred to as an indirect interstitial mechanism, in which the hydride ion is transformed to a proton, by donating two extra electrons to Ti ions, and then diffuses by interstitial diffusion. We found that in $\mathrm{BaTiO}_{3}$ the required energy for this process is $2.4 \mathrm{eV}$, which, in agreement with previous results, ${ }^{7,8}$ is considerably larger than the typical activation energy for hydride-ion conduction in this system $(<0.5 \mathrm{eV}) .^{\mathbf{4}, \mathbf{8}}$ Thus, we have no reason to expect that the indirect interstitial mechanism takes place in $\mathrm{BaTiO}_{3}$, which is in full accordance with the absence of any signature of $\mathrm{O}-\mathrm{H}$ species in the vibrational spectra.
The comparison of the experimental results with the results from DFT calculations show that the vibrational spectra can be largely divided into three different regions; a low-energy $(<90$ $\mathrm{meV}$ ) region related to lattice vibrations, a medium-energy (90$150 \mathrm{meV}$ ) region related to local hydride ion vibrations, and a higher energy (>150 meV) region related to higher-order transitions. Specifically, the INS spectra for all three samples are characterized by two intense bands at approximately 114 $\left(\omega_{\perp}\right)$ and $128\left(\omega_{\|}\right) \mathrm{meV}$, which are assigned to fundamental Ti$\mathrm{H}$ vibrational modes perpendicular and parallel and to the $\mathrm{Ti}-$ $\mathrm{H}-\mathrm{Ti}$ bond direction, respectively. Additionally, the INS spectra contain four weaker bands, at approximately $99\left(\omega_{1}\right), 110\left(\omega_{2}\right)$, $137\left(\omega_{3}\right)$ and $145\left(\omega_{4}\right) \mathrm{meV}$, as a result of small differences in the local coordination of the hydride ions in the materials.

When comparing these experimental results with the DFT calculations, one should note that the use of the PBE functional generally underestimates the vibrational frequencies by about 7 $\mathrm{meV}$ for $\omega_{\perp}$ and $2 \mathrm{meV}$ for $\omega_{\|}$, compared with the more accurate HSE functional. ${ }^{13}$ With this discrepancy taken into account, the calculated frequencies of around $\omega_{\perp}=104-106 \mathrm{meV}$ and $\omega_{\|}=$ 124-127 meV for both the $\mathrm{H}_{\mathrm{O}} \mathrm{V}_{\mathrm{O}}^{\times(2)}$ and $\mathrm{H}_{\mathrm{O}} \mathrm{v}_{\mathrm{O}}^{\times(3)}$ configuration, as well as the bandstate configuration $\mathrm{H}_{\mathrm{O}}^{\times}$(band), are in excellent agreement with the experimental values of $\omega_{\perp}=114 \mathrm{meV}$ and $\omega_{\|}=128 \mathrm{meV}$. The exception is the $\mathrm{H}_{\mathrm{O}} \mathrm{v}_{\mathrm{O}}^{\times(1)}$ configuration, which is characterized by a hydride ion and an oxygen vacancy as first nearest neighbour and for which the $\omega_{\perp}^{(2)}$ mode is downward-shifted to $93 \mathrm{meV}$ in the calculations. Considering an underestimation of $7 \mathrm{meV}$ of the frequency of this band, it matches almost perfectly with the frequency of the $99\left(\omega_{1}\right) \mathrm{meV}$ band in the experimental spectrum. From this we infer that the positioning of the oxygen vacancy with respect to the hydride ion has only a small effect on the vibrational spectra, except when the oxygen vacancy is first nearest neighbour to the hydride ion. Furthermore, we note that the highest-frequency band at approximately $145\left(\omega_{4}\right)$ meV is in very good agreement with the frequency of the $\omega_{\|}$mode for the polaron state. It follows that, even though the bandstate configuration is the dominating configuration of the hydride ions, our results tentatively indicate the presence of a non-negligible amount of electron polarons, at least in the sample CA2.

The new insight into the local structure and vibrational dynamics of the metal hydride reduced $\mathrm{BaTiO}_{3}$ samples, containing the simultaneous presence of hydride ions and oxygen vacancies, can be linked to the nature of diffusional dynamics of the hydride ions. In our previous work, we investigated the nature of diffusional hydride ion dynamics in CA1, CA2, and NAB, by QENS techniques. Analyses of QENS spectra measured at low $(T=225$ and $250 \mathrm{~K})$ and high $(T=400-700 \mathrm{~K})$ temperature show that the dynamics can be adequately described by established models of jump diffusion. At low temperature, $T \leq$ $250 \mathrm{~K}$, all of the models feature a characteristic jump distance of about $2.8 \AA$, thus of the order of the distance between neighboring oxygen atoms or oxygen vacancies of the perovskite structure and a mean residence time between successive jumps of the order of $0.1 \mathrm{~ns}$. At higher temperatures, $>400 \mathrm{~K}$, the jump distance increases to about $4 \AA$, thus of the order of the distance between next-nearest neighboring oxygen atoms or oxygen 
vacancies, with a mean residence time of the order of picoseconds. Our present results confirm the existence of these specific local structural configurations and hence support these previous findings on the mechanism of hydride ion transport in perovskite type oxyhydrides based on $\mathrm{BaTiO}_{3}$.

Further insight into the nature of the hydride ion sites comes from the analysis of the MSD of the hydride ions. Crucially, the combined analyses of INS and DFT data show that hydrogen vibrational motions are very localized in nature and only very weakly affected by the dynamics of the surrounding perovskite structure.

\section{Conclusions}

We investigated the local coordination environment of hydride ions in three metal hydride reduced $\mathrm{BaTiO}_{3}$ samples characterized by the simultaneous presence of hydride ions and oxygen vacancies, using a combination of powder X-ray diffraction, thermal gravimetric analysis, nuclear magnetic resonance, inelastic neutron scattering, and density functional theory calculations. We show that the hydride ions are located on oxygen positions of the perovskite structure and that the hydride ion vibrational dynamics are characterized by two main bands, at $114 \mathrm{meV}\left(\omega_{\perp}\right)$ and $128\left(\omega_{\|}\right) \mathrm{meV}$, respectively, assigned as $\mathrm{Ti}-\mathrm{H}$ vibrational modes perpendicular and parallel to the Ti$\mathrm{H}-\mathrm{Ti}$ bond direction, and four additional, weaker, bands near the $\omega_{\perp}$ and $\omega_{\|}$bands, associated with variations of the local coordination environment of the hydride ions in the materials.

Crucially, the combined analyses of INS and DFT data confirm the presence of both nearest neighbour and nextnearest neighbouring oxygen vacancies to the hydride ions. This supports previous findings from quasielastic neutron scattering experiments, that the hydride ion transport is governed by jump diffusion dynamics between neighbouring and next-nearest neighbouring hydride ion-oxygen vacancy configurations. Analysis of the MSD of the hydride ions, as determined both experimentally and theoretically, confirms that the hydrogen vibrational motions are very localized in nature and only very weakly affected by the dynamics of the surrounding perovskite structure.

\section{Author contributions}

C. E.-Ö., L. M. and M. K. performed and interpreted the INS experiments, assisted by S. F. P. and N. H. J. The DFT simulations were performed and interpreted by E. J. G., under guidance of G. W. The NMR spectroscopy experiments were performed and interpreted by A. J., under guidance of A. J. P. The samples were synthesized by R. N., under guidance of U. H. The study was designed by M. K., together with U. H. and G. W. All authors provided critical comments on the work and to the writing of the paper.

\section{Conflicts of interest}

There are no conflicts to declare.

\section{Acknowledgements}

This research was funded by the Swedish Foundation for Strategic Research (grant no. ICA10-0001), the Swedish Research Council (grant no. 2014-3980 and 2016-03441) and the Swedish Energy Agency (grant no. 36645-1 and no. 45410-1). A portion of this research used resources at the Spallation Neutron Source, a DOE Office of Science User Facility operated by the Oak Ridge National Laboratory. The STFC is thanked for access to neutron beam facilities.

\section{References}

1 Y. Kobayashi, O. J. Hernandez, T. Sakaguchi, T. Yajima, T. Roisnel, Y. Tsujimoto, M. Morita, Y. Noda, Y. Mogami, A. Kitada, M. Ohkura, S. Hosokawa, Z. Li, K. Hayashi, Y. Kusano, J. eun Kim, N. Tsuji, A. Fujiwara, Y. Matsushita, K. Yoshimura, K. Takegoshi, M. Inoue, M. Takano and H. Kageyama, Nat. Mater., 2012, 11, 507-511.

2 T. Sakaguchi, Y. Kobayashi, T. Yajima, M. Ohkura, C. Tassel, F. Takeiri, S. Mitsuoka, H. Ohkubo, T. Yamamoto, J. Kim, N. Tsuji, A. Fujihara, Y. Matsushita, J. Hester, M. Avdeev, K. Ohoyama and H. Kageyama, Inorg. Chem., 2012, 51, 11371-11376.

3 T. Yamamoto, R. Yoshii, G. Bouilly, Y. Kobayashi, K. Fujita, Y. Kususe, Y. Matsushita, K. Tanaka and H. Kageyama, Inorg. Chem., 2015, 54, 1501-1507.

4 C. Eklöf-Österberg, R. Nedumkandathil, U. Häussermann, A. Jaworski, A. J. Pell, M. Tyagi, N. Jalarvo, B. Frick, A. Faraone and M. Karlsson, J. Phys. Chem. C, 2019, 123, 2019-2030.

5 Y. Tang, Y. Kobayashi, K. Shitara, A. Konishi, A. Kuwabara, T. Nakashima, C. Tassel, T. Yamamoto and H. Kageyama, Chem. Mater., 2017, 29, 8187-8194.

6 F. Takeiri, K. Aidzu, T. Yajima, T. Matsui, T. Yamamoto, Y. Kobayashi, J. Hester and H. Kageyama, Inorg. Chem., 2017, 56, 13035-13040.

7 X. Liu, T. S. Bjørheim, L. Vines, Ø. S. Fjellvåg, C. Granerød, Ø. Prytz, T. Yamamoto, H. Kageyama, T. Norby and R. Haugsrud, J. Am. Chem. Soc., 2019, 141, 4653-4659.

8 X. Liu, T. Bjørheim and R. Haugsrud, J. Mater. Chem. A, 2017, 5, 1050-1056.

9 J. Zhang, G. Gou and B. Pan, J. Phys. Chem. C, 2014, 118, 17254-17259.

10 Y. Iwazaki, T. Suzuki and S. Tsuneyuki, J. Appl. Phys., 2010, 108, 083705.

11 T. Yajima, A. Kitada, Y. Kobayashi, T. Sakaguchi, G. Bouilly, S. Kasahara, T. Terashima, M. Takano and H. Kageyama, J. Am. Chem. Soc., 2012, 134, 8782-8785.

12 G. Bouilly, T. Yajima, T. Terashima, W. Yoshimune, K. Nakano, C. Tassel, Y. Kususe, K. Fujita, K. Tanaka, T. Yamamoto, Y. Kobayashi and H. Kageyama, Chem. Mater., 2015, 27, 6354-6359.

13 E. Jedvik Granhed, A. Lindman, C. Eklöf-Österberg, M. Karlsson, S. F. Parker and G. Wahnström, J. Mater. Chem. A, 2019, 7, 16211-16221. 
14 A. J. Pell and G. Pintacuda, Prog. Nucl. Magn. Reson. Spectrosc., 2015, 84-85, 33-72.

15 A. J. Pell, G. Pintacuda and C. P. Grey, Prog. Nucl. Magn. Reson. Spectrosc., 2019, 111, 1-271.

16 T.-L. Hwang, P. Van Zijl and M. Garwood, J. Magn. Reson., 1998, 133, 200-203.

17 G. Kervern, G. Pintacuda and L. Emsley, Chem. Phys. Lett., 2007, 435, 157-162.

18 ISIS - Merlin, http://www.isis.stfc.ac.uk/instruments/merlin.

19 R. Bewley, R. Eccleston, K. McEwen, S. Hayden, M. Dove, S. M. Bennington, J. Treadgold and R. Coleman, Phys. B, 2006, 385-386, 1029-1031.

20 S. F. Parker, F. Fernandez-Alonso, A. J. Ramirez-Cuesta, J. Tomkinson, S. Rudic, R. S. Pinna, G. Gorini and J. Fernández Castañon, J. Phys.: Conf. Ser., 2014, 554, 012003.

21 S. F. Parker, C. J. Carlile, T. Pike, J. Tomkinson, R. J. Newport, C. Andreani, F. P. Ricci, F. Sachetti and M. Zoppi, Phys. B, 1998, 241-243, 154-156.

22 web-page: https://neutrons.ornl.gov/vision.

23 O. Arnold, et al., Nucl. Instrum. Methods Phys. Res., Sect. A, 2014, 764, 156-166.

24 R. Coldea, ISIS, Rutherford Appleton Laboratory, Mslice, http://mslice.isis.rl.ac.uk/MainPage/, 2004.

25 R. Azuah, L. Kneller, Y. Qiu, P. L. W. Tregenna-Piggott, C. Brown, J. Copley and R. Dimeo, J. Res. Natl. Inst. Stand. Technol., 2009, 114, 341-358.

26 V. F. Sears, Neutron News, 1992, 3, 26-37.
27 P. C. H. Mitchell, S. F. Parker, A. Ramirez-Cuesta, and J. Tomkinson, Vibrational Spectroscopy with Neutrons, Word Scientific, Singapore, 2005.

28 L. Mazzei, A. Perrichon, A. Mancini, G. Wahnström, L. Malavasi, S. F. Parker, L. Börjesson and M. Karlsson, J. Mater. Chem. A, 2019, 7, 7360-7372.

29 J. Tomkinson, S. F. Parker and D. Lennon, J. Chem. Phys., 2010, 133, 034508.

30 S. F. Parker, S. M. Bennington, A. J. Ramirez-Cuesta, G. Auffermann, W. Bronger, H. Herman, K. P. J. Williams and T. Smith, J. Am. Chem. Soc., 2003, 125, 11656-11661.

31 P. E. Blöchl, Phys. Rev. B: Condens. Matter Mater. Phys., 1994, 50, 17953-17979.

32 G. Kresse and D. Joubert, Phys. Rev. B: Condens. Matter Mater. Phys., 1999, 59, 1758-1775.

33 G. Kresse and J. Furthmüller, Comput. Mater. Sci., 1996, 6, 15-50.

34 G. Kresse and J. Furthmüller, Phys. Rev. B: Condens. Matter Mater. Phys., 1996, 54, 11169-11186.

35 J. P. Perdew, K. Burke and M. Ernzerhof, Phys. Rev. Lett., 1996, 77, 3865-3868.

36 A. Togo and I. Tanaka, Scr. Mater., 2015, 108, 1-5.

37 R. Nedumkandathil, A. Jaworski, J. Grins, D. Bernin, M. Karlsson, C. Eklöf-Österberg, A. Neagu, C.-W. Tai, A. Pell and U. Häussermann, ACS Omega, 2018, 3, 1142611438. 